\title{
ARTIGO
}

\section{DESENVOLVIMENTO DE FRAMEWORK PARA ELABORAÇÃO DE RANKINGS E ANÁLISES DOS RELATÓRIOS DE SUSTENTABILIDADE DE COMPANHIAS INTEGRADAS DE ÓLEO E DE GÁS COM ABORDAGEM NO GRI ${ }^{1}$}

\author{
Ary Branco Adurens Júnior ${ }^{2}$ \\ Eduardo Christiano Cecone \\ Chang Chung Wei \\ Fernando Gasi \\ Douglas Alves Cassiano
}

\begin{abstract}
RESUMO
O Global Report Initiative (GRI) é uma organização internacional, sem fins lucrativos, que disponibiliza dados de emissões e diretrizes para elaboração de relatórios de impacto na sustentabilidade em diversos segmentos industriais, como o setor de óleo e de gás. Entretanto, limita-se à disponibilização dos dados, não procedendo outras análises, no sentido de produzir informações mais elaboradas, como por exemplo, o estabelecimento de benchmarks. Neste sentido, o presente trabalho propõe um framework, visando a organizar as informações na elaboração de rankings das companhias integradas de óleo e gás, verificando-se as relações existentes entre informações de produção e emissões de gases, utilizando uma correlação produto-momento. Como resultado específico, obtiveram-se rankings relativos à produção e indicadores de emissões das companhias integradas de óleo e gás. Infere-se do estudo que as empresas deste segmento energético mais produtivas também são as que mais provocam emissões, e também que empresas de origem europeia apresentam tendência a produzirem menos emissões que empresas originárias de outras partes do mundo. Com a análise das informações decorrentes do ranking obtido da aplicação do framework proposto, há a possibilidade de as empresas integradas do segmento de óleo e gás planejarem de forma mais assertiva suas atividades, no que tange a um posicionamento sustentável por parte delas.
\end{abstract}

Palavras-chave: Emissões gasosas; Companhias integradas de óleo e gás; Ranqueamento. Global Report Initiative.

\section{INTRODUÇÃO}

\subsection{O GRI e seus Relatórios de Sustentabilidade}

\footnotetext{
${ }^{1}$ Como citar este artigo: ADURENS JÚNIOR, Ary Branco; CECONE, Eduardo Christiano; WEI, Chang Chung; GASI, Fernando; CASSIANO, Douglas Alves. Desenvolvimento de framework para elaboração de rankings e análises dos relatórios de sustentabilidade de companhias integradas de óleo e de gás com abordagem no GRI. ForScience: revista científica do IFMG, Formiga, v. 7, n. 2, e00299, jul./dez. 2019. DOI: 10.29069/forscience.2019v7n2.e299.
}

\footnotetext{
${ }^{2}$ Autor para correspondência: Ary Branco Adurens Júnior, e-mail: adurens@ gmail.com.
} 
A Global Report Initiative (GRI) é uma organização internacional, independente, multistakeholder e sem fins lucrativos, a qual, desde 1997, oferece apoio a empresas, governos e outras organizações no sentido de comunicar o impacto dos negócios destas em algumas das dimensões da sustentabilidade ambiental (CHEN et al., 2014). Esse suporte se dá principalmente no estabelecimento de padrões e de diretrizes para estruturação e criação de relatórios de sustentabilidade, assim como na divulgação de dados de emissões.

As diretrizes da GRI aplicam-se a organizações multinacionais, agências públicas, pequenas e médias empresas, ONGs e grupos industriais, dentre outros. Somente em 2015, mais de 7500 organizações utilizaram as diretrizes da GRI para elaborar relatórios de sustentabilidade próprios (CNBC, 2016).

Os relatórios de sustentabilidade tornaram-se elementos chave nas organizações, sendo importantes instrumentos na prestação de contas empresariais a stakeholders, clientes, parceiros e a sociedade (DOMINGUESet al., 2017). Em pesquisa realizada por Ligteringen e Arbex (2012), as principais motivações que as organizações apresentam em comum para embarcarem em um processo de produzir relatos de sustentabilidade são:

- Demonstrar compromisso e transparência;

- Demonstrar capacidade de participar em mercados competitivos;

- Planejar suas atividades para posicionamento da empresa como sustentável;

- Obedecer à legislação.

Ainda segundo estes mesmos autores, há duas classes de valores e ganhos adicionais às organizações durante $\mathrm{o}$ processo de preparação e elaboração dos relatos de sustentabilidade: os valores das mudanças internas e os valores de reconhecimento:

1. Valores das Mudanças Internas:

- Desenvolvimento de visão e estratégia;

- Melhoria nos sistemas de gestão, aperfeiçoamento dos processos internos e estabelecimento de metas;

- Identificação de pontos fortes e fracos;

- Atração e retenção de funcionários;

- Integração entre departamentos e estímulo à inovação;

- Conscientização do Conselho de Administração; 
- Vantagem competitiva e liderança;

- Atração de investidores.

2. Valores de Reconhecimento:

- Fortalecimento da reputação, conquista de confiança e respeito;

- Transparência e diálogo com stakeholders;

- Demonstração de compromisso com a sustentabilidade;

- Comparabilidade e benchmarking.

\subsection{Empresas Integradas de Óleo e de Gás e suas Emissões Gasosas}

A denominação "companhia integrada" no segmento industrial de óleo e gás refere-se à entidade empresarial deste setor que executa as atividades que vão desde a exploração, produção e refino até a distribuição e comercialização de seus produtos (WANG; LI, 2017).

Devido ao grau de complexidade decorrente de suas várias atividades de operações, as empresas integradas efetuam uma classificação dos segmentos de sua cadeia produtiva em upstream (exploração, desenvolvimento e produção), midstream (transporte, estocagem e processamento) e downstream (refino, transporte para o mercado e comercialização). Além destas atividades, as empresas integradas de óleo e gás, podem possuir atividades adicionais, como: geração e comercialização de energia, produção de fertilizantes, biocombustíveis, produção de combustíveis sintéticos a partir do gás natural (GTL - gas to liquids) ou carvão (CTL - coal to liquids) e produtos petroquímicos.

Dentre as várias fontes de emissão de gases de efeito estufa (GEE), a produção de energia é a principal causadora de emissão destes gases (RAHMAN; MIAH,2017). Em se tratando de emissões de GEE, especificamente na indústria de óleo e gás, as emissões relativas às suas operações representam em média 15\% (upstream, midstream e downstream), enquanto que $85 \%$ das emissões são efetuadas pela queima dos combustíveis produzidos a partir do óleo e gás (EUROPIA, 2011).

Dentro do contexto de demonstrar a sustentabilidade de suas operações, as empresas do setor de óleo e gás seguem as seguintes diretrizes desenvolvidas por associações do setor: IOGP (International Oil and Gas Producers), API (American Petroleum Institute) e IPIECA (The global oil and gas industry association for environmental and social issues); o uso dessas diretrizes têm o objetivo de reportar de forma voluntária as atividades, os impactos e a 
evolução ao longo de cada ano, conforme as orientações do Oil and Gas industry guidance on voluntary sustainability reporting.

No tocante às novas legislações na busca da redução das emissões, destaca-se a iniciativa de países europeus para a redução das emissões de gases de efeito estufa em $20 \%$ até 2020, com base nas emissões de 1990 (BENITES-LAZARO; MELLO-THÉRY, 2017).

Uma das políticas envolvidas para o alcance deste objetivo pela união européia é o EU ETS ou Emissions Trading System (SKJAERSETH; WETTESTAD, 2013). Nos Estados Unidos, ação similar foi adotada por meio do sistema RGGI ou Regional Greenhouse Gas Initiative (PERDAN; AZAPAGIC, 2011).

O IOGP, API e IPIECA, reconhecem a importância e o alcance que o GRI possui perante uma série de stakeholders. Isso pode ser percebido no fato de que os membros da IPIECA, IOGP e API, responsáveis pelo desenvolvimento do Oil and Gas industry guidance on voluntary sustainability reporting, procuram trabalhar de forma integrada com os membros do GRI. Tal integração é ressaltada pela correspondência e alta aderência apresentada entre os indicadores utilizados pelas diretrizes do IPIECA, API e IOGP e pela metodologia do GRI (IPIECA/API/IOGP, 2015).

Entretanto, diversos autores têm questionado a eficácia dos relatórios do GRI, principalmente no que concerne ao uso e à aplicação das informações nas empresas e na sociedade como operadores de mudanças efetivamente sustentáveis (VIGNEAU et al., 2015; COFFEY, 2016; DOMINGUES et al., 2017). Algumas das causas pontuadas são a confecção dos relatórios meramente com a finalidade de serem instrumento publicitário sem o comprometimento em ações efetivas nas organizações da carência de análises mais aprofundadas das informações ( MARIMON et al., 2012; SANTANA; CASSIANO; LOURENÇO, 2015; ABREU, 2016).

\subsection{Frameworks}

Existem diversas maneiras distintas para se definir o que é um framework. Porém, muitos autores como Govoni (1999) e Fayad, Schmidt e Johnson (1999) propõem uma definição a partir do ponto de vista da aplicação do framework, ou seja, como sendo uma coleção de padrões dedicados a resolver problemas por meio de uma sequência de ações sistematizadas.

Para haver essa sistematização de ações direcionadas à solução, as aplicações escolhidas devem possuir características comuns que as definam especificamente, como 
pertencer a uma mesma classe ou domínio de problemas. Esta sequência de ações é usualmente denominada arquitetura e, apesar de ser planejada para prover uma solução de problemas com características bem específicas, pode, dependendo do contexto e condições de contorno, ser flexível e extensível (MURADIAN et al., 2009; GJORUP et al., 2016).

Embora o termo framework tenha sido utilizado inicialmente por áreas do conhecimento relacionadas à tecnologia da informação, atualmente teve seu uso estendido posteriormente a outras áreas de conhecimento, como nas relacionadas ao ramo de administração, ciências sociais, e ambientais, dentre outras.

\subsection{Objetivo e justificativa da pesquisa}

O presente trabalho tem por objetivo elaborar um framework para criação de rankings de emissões em função da produtividade de empresas do setor energético de óleo e de gás, baseado nos dados reportados nos relatórios do GRI.

A motivação para execução deste trabalho foi a constatação da lacuna existente na simples disponibilização dos dados efetuada pelos relatórios do GRI, sem que se efetuem análises mais elaboradas que possibilitem transformar estes dados em informações de cunho gerencial, como na elaboração de rankings ou benchmarks.

\section{METODOLOGIA}

O framework proposto no presente trabalho de pesquisa consiste numa arquitetura cujo desenvolvimento segue de forma sintética as seguintes etapas: prospecção das empresas de óleo e de gás integradas; seleção dos indicadores avaliados; obtenção dos valores dos indicadores escolhidos para cada empresa e análise dos dados para elaboração de rankings, conforme apresentado na Figura 1. 


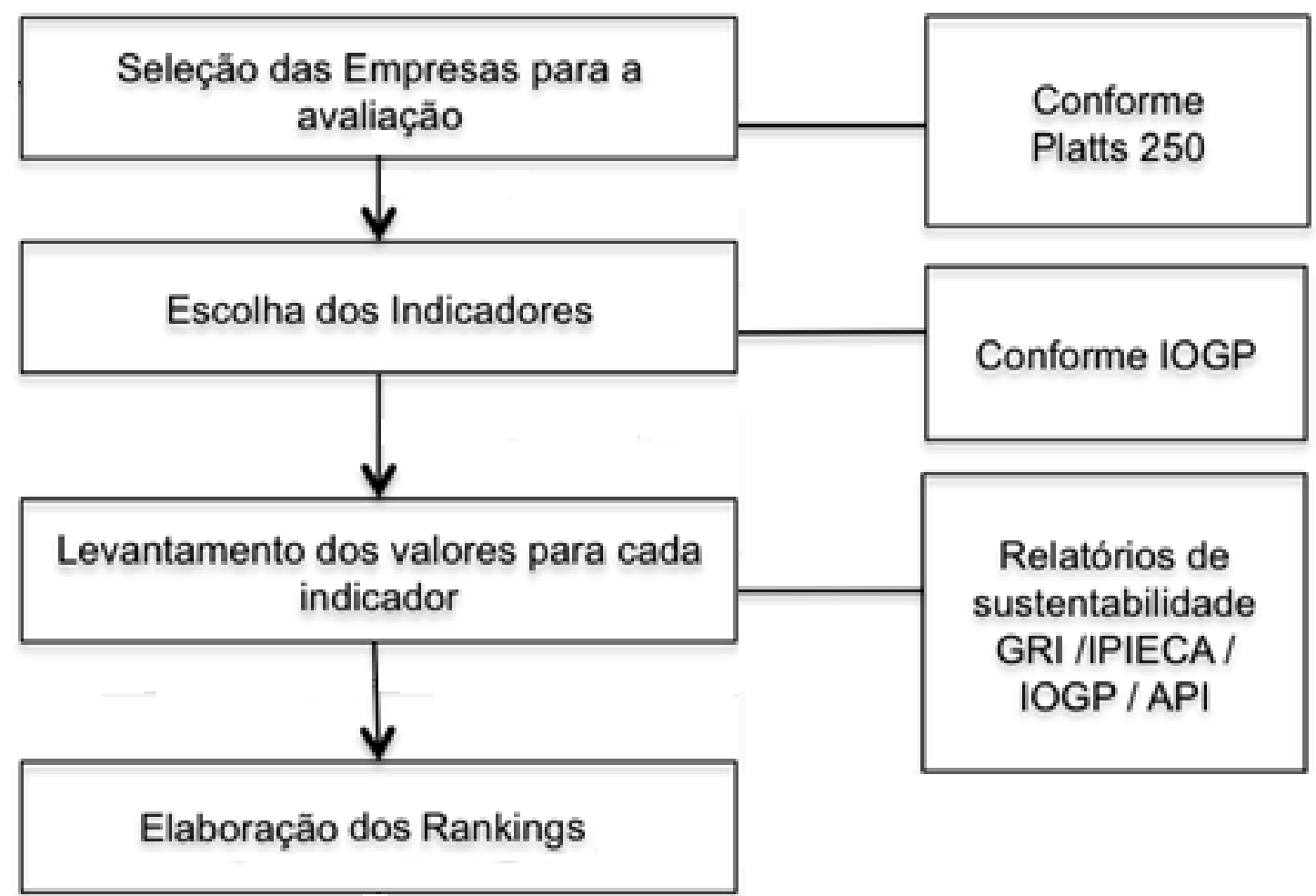

Figura 1 - Framework proposto no trabalho Fonte: Elaboração própria.

Embora sigam as diretrizes propostas pelo GRI na elaboração de seus relatórios de sustentabilidade, as informações necessárias para o desenvolvimento desta pesquisa estão disponíveis em instituições e bases de dados distintas, que não operam de forma integrada. Assim, uma prospecção das informações em cada instituição em suas bases de dados foi necessária para o desenvolvimento da pesquisa.

$\mathrm{Na}$ etapa de seleção de empresas inicialmente foi obtida uma listagem com trinta empresas de óleo e de gás integradas, consultando-se a lista das empresas da indústria de óleo e de gás elaborada pela plataforma Platts, por meio do ranking TOP 250 (PLATTS, 2015).

Como indicador de produção, foi adotado o valor de produção total reportado de cada companhia. Em relação aos grupos de indicadores deste trabalho referentes às emissões gasosas, ressalta-se que eles foram escolhidos pelas orientações dispostas no relatório Environmental Performance Indicators, o qual é elaborado anualmente pela International Association of Oil \& Gas Producers (IPIECA/API/IOGP, 2015).

Especificamente em relação ao grupo de emissões gasosas, vale ressaltar que estão inclusos os seguintes indicadores segundo o IOGP: Emissões de $\mathrm{CO}_{2}$; Emissões de $\mathrm{CH}_{4}$; Emissões de GHG (greenhouse gases, $\mathrm{CO}_{2}+\mathrm{CH}_{4}$, expressos em milhões de $\mathrm{CO}_{2 \text { equivalente); }}$ ) Emissões de $\mathrm{SO}_{\mathrm{x}}$; Emissões de $\mathrm{NO}_{\mathrm{x}}$ e Emissões de compostos orgânicos voláteis (VOC's). 
Emissões de GHG são obtidas pelo somatório das emissões de $\mathrm{CO}_{2}$ e $\mathrm{CH}_{4}$, logo, considerouse como indicador a ser utilizado neste estudo somente as emissões de GHG.

Efetuada a escolha de quais indicadores seriam avaliados, a etapa seguinte constitui-se no levantamento dos valores para cada indicador.

Os dados para os indicadores escolhidos foram obtidos pela consulta dos relatórios de sustentabilidade disponíveis no GRI, emitidos pelas empresas de óleo e gás integradas em 2015; dados esses, relativos às suas respectivas operações do ano base de 2014 (BG, 2015; BP, 2015; CHEVRON, 2015; ECOPETROL, 2015; ENI, 2015; EXXON, 2015; GRI, 2015; HESS, 2015; OMV, 2015; PETROBRAS, 2015; REPSOL, 2015; SHELL, 2015; STATOIL, 2015; SUNCOR, 2015; TOTAL, 2015).

Cabe ressaltar, entretanto, que, apesar de haver boas práticas e normas estabelecidas para a confecção dos relatórios do GRI, não há uma padronização absoluta no que se refere às informações divulgadas. Desse modo, há empresas que não apresentam as informações completas ou referentes a todos os indicadores. Nestes casos, a carência de informações consistentes constitui-se uma condição de contorno do framework proposto, e estas empresas acabam sendo descartadas da lista de companhias integradas avaliadas.

A última etapa do framework proposto é o estabelecimento dos rankings, a partir das informações tabuladas para as empresas integradas de óleo e de gás. Nesta pesquisa, estabeleceram-se rankings relativos à produção e a emissões das companhias.

O ranking de produção das empresas deste setor energético foi confeccionado diretamente a partir das informações disponibilizadas nos relatórios. Para obtenção dos rankings relativos às emissões por produção, obteve-se uma normalização das companhias pela razão entre cada um dos indicadores de emissões totais (GHG, NOx, SOx e VOC's) e a respectiva produção total $(P)$ de cada companhia, mantendo a classificação das empresas por ordem decrescente de produção.

Por fim, as variáveis dos rankings obtidos foram avaliadas em relação à produção de óleo e de gás das companhias, utilizando-se o coeficiente de correlação produto-momento, também denominado coeficiente de correlação de Pearson $\left(\rho_{X_{2} Y}\right)$. Este coeficiente de correlação avalia a relação linear entre duas variáveis aleatórias $(X, Y)$, dividindo-se a covariância destas pelo produto de seus desvios padrão, conforme demonstrado na equação 1 .

$$
\rho_{x, y}=\frac{\operatorname{cov}\left(X_{Y} Y\right)}{\sigma_{X} \sigma_{Y}}
$$


O valor do coeficiente de correlação de Pearson pode apresentar valores entre -1 a $1 . \mathrm{O}$ sinal positivo indica existência de uma relação diretamente proporcional, sendo o sinal negativo uma indicação de relação inversamente proporcional.

Já a interpretação do valor sugere a força da relação entre as variáveis, pois, independentemente do sinal positivo ou negativo, a faixa de valores do coeficiente entre 0 e 0,29 indica uma correlação desprezível; já a faixa entre 0,30 e 0,49 pode ser considerada uma correlação moderada e para valores maiores que 0,50 a correlação pode ser interpretada como sendo forte (COHEN, 2002).

\section{RESULTADOS E DISCUSSÕES}

A partir da listagem inicial das trinta companhias integradas de óleo e gás selecionadas de acordo com os critérios adotados para o framework proposto, foi verificada a disponibilidade dos dados de cada indicador para cada empresa utilizando relatórios de sustentabilidade próprios de cada uma. Ao término desta análise, dezesseis empresas foram descartadas da lista, pois não apresentavam a totalidade das informações dos indicadores de emissões. A Tabela 1 apresenta as informações disponibilizadas pelas empresas, de acordo com o ranking na ordem decrescente de produção total $(P)$ de cada uma delas.

Tabela 1- Ranking de produção das companhias integradas de óleo e de gás

\begin{tabular}{cccccc}
\hline Empresa & $\begin{array}{c}\text { Produção } \\
\left(10^{6} \mathrm{GJ}\right)\end{array}$ & $\begin{array}{c}\text { GHG } \\
\left(10^{6} \text { ton }\right)\end{array}$ & $\begin{array}{c}\text { NOx } \\
\left(10^{3} \text { ton }\right)\end{array}$ & $\begin{array}{c}\text { SOx } \\
\left(10^{3} \text { ton }\right)\end{array}$ & $\begin{array}{c}\text { VOC's } \\
\left(10^{3} \text { ton }\right)\end{array}$ \\
\hline Exxon & 8491 & 122 & 140 & 100 & 200 \\
BP & 6741 & 55 & 130 & 39 & 98 \\
Shell & 6632 & 76 & 146 & 97 & 151 \\
Petrobras & 5710 & 81 & 299 & 133 & 249 \\
Chevron & 5501 & 56 & 139 & 113 & 135 \\
Total & 4591 & 48 & 93 & 65 & 80 \\
ENI & 3419 & 44 & 90 & 25 & 28 \\
Statoil & 2133 & 17 & 47 & 2 & 72 \\
Ecopetrol & 1616 & 8 & 23 & 17 & 108 \\
BG & 1297 & 8 & 35 & 26 & 9 \\
Suncor & 1144 & 20 & 28 & 29 & 18 \\
Repsol & 758 & 15 & 35 & 30 & 46
\end{tabular}




\begin{tabular}{cccccc} 
Hess & 704 & 6 & 10 & 2 & 13 \\
OMV & 661 & 13 & 15 & 3 & 16 \\
\hline
\end{tabular}

Fonte: Elaboração própria.

$\mathrm{Na}$ Tabela 2, são demonstrados os indicadores obtidos pela razão entre emissões e produção, de acordo com a posição de cada empresa no ranking apresentado na Tabela 1.

Tabela 2 - Indicadores de emissões de acordo com a posição no ranking de produção das companhias integradas de óleo e de gás

\begin{tabular}{ccccc}
\hline Empresa & $\begin{array}{c}\mathrm{GHG} / \mathrm{P} \\
\left(10^{6} \text { ton } / 10^{6} \mathrm{GJ}\right)\end{array}$ & $\begin{array}{c}\mathrm{NOx} / \mathrm{P} \\
\left(10^{3} \text { ton } / 10^{6} \mathrm{GJ}\right)\end{array}$ & $\begin{array}{c}\mathrm{SOx} / \mathrm{P} \\
\left(10^{3} \text { ton } / 10^{6} \mathrm{GJ}\right)\end{array}$ & $\begin{array}{c}\text { VOC's/P } \\
\left(10^{3} \text { ton } / 10^{6} \mathrm{GJ}\right)\end{array}$ \\
\hline Exxon & 0,0144 & 0,0165 & 0,0118 & 0,0236 \\
BP & 0,0065 & 0,0153 & 0,0046 & 0,0115 \\
Petrobras & 0,0090 & 0,0172 & 0,0114 & 0,0178 \\
Chevron & 0,0095 & 0,0352 & 0,0157 & 0,0293 \\
Total & 0,0057 & 0,0164 & 0,0133 & 0,0159 \\
ENI & 0,0052 & 0,0110 & 0,0077 & 0,0094 \\
Statoil & 0,0020 & 0,0106 & 0,0029 & 0,0033 \\
Ecopetrol & 0,0009 & 0,0055 & 0,0002 & 0,0085 \\
BG & 0,0009 & 0,0027 & 0,0020 & 0,0127 \\
Suncor & 0,0024 & 0,0041 & 0,0031 & 0,0011 \\
Repsol & 0,0018 & 0,0033 & 0,0034 & 0,0021 \\
Hess & 0,0007 & 0,0041 & 0,0035 & 0,0054 \\
OMV & 0,0015 & 0,0012 & 0,0002 & 0,0015 \\
\hline
\end{tabular}

Fonte: Elaboração própria.

A partir dos valores apresentados na Tabela 2, é possível estimar os valores dos coeficientes de correlação de Pearson. Foram obtidos os valores de coeficientes de 0,9436; 0,7776; 0,7949 e 0,7959 respectivamente para os indicadores GHG/P; Nox/P; SOx/P e VOC's/ $P$, os quais foram normalizados em relação à produção de óleo e de gás das companhias.

Tais valores positivos demonstram uma forte correlação de ordem direta entre produção e emissões. Estes resultados confirmam que, quanto mais uma empresa produz óleo e gás, mais emissões também produzirá, quantificando essa relação em termos de dependência 
linear destas variáveis. Portanto, depreende-se desta análise que, quanto menor a produção de uma empresa, e consequentemente menor a complexidade de suas operações, menores serão seus índices de emissões.

Analisando a Tabela 2, também é possível inferir que as empresas oriundas de países europeus, como a Statoil, BG, Repsol e OMV dentre outras, tendem a apresentar menores índices de emissões. No entanto, não foi objeto de estudo deste trabalho e careceria de uma pesquisa direcionada especificamente a fim de elucidar esta questão, porém este fato ocorre possivelmente em atendimento às legislações mais rígidas existentes na Europa referentes às emissões de poluentes. As exceções que apareceram neste estudo em relação a baixas emissões são a colombiana Ecopetrol, a canadense Suncor e a Norte Americana Hess, que também apresentam baixos índices de emissões relativos, mesmo não sendo empresas da Europa.

\section{CONCLUSÃO}

A metodologia elaborada na forma de um framework próprio resultou no estabelecimento em rankings de produção, correlacionando-se com as emissões das empresas de óleo e gás integradas, a partir das informações reportadas nos relatórios do GRI e de demais bases de dados mencionadas.

Vale ressaltar novamente sobre o framework proposto, o qual, apesar de constituir uma referência mundial em se tratando de relatórios empresariais de sustentabilidade, não apresenta uma padronização absoluta para as informações disponibilizadas sobre emissões nos relatórios de sustentabilidade com abordagem no GRI, fato este que acarretou uma condição de contorno significativa na seleção das empresas integradas do setor de óleo e de gás.

Foram obtidos dois rankings das companhias integradas avaliadas pelo framework proposto, referentes à produção total das empresas e indicadores de emissões gasosas por produção, este último também ordenado de acordo com a produção das empresas. Infere-se que há uma relação significativa e diretamente proporcional entre a produção de óleo e gás e emissões por parte das empresas integradas deste setor energético, de acordo com os valores de coeficientes produto-momento obtidos, respectivamente: 0,9436; 0,7776;0,7949 e 0,7959 para os indicadores GHG/P; Nox/P; SOx/P e VOC's/P, em relação à produção total. 
Deste resultado, infere-se que possivelmente quanto menor a produção e consequentemente a complexidade de operações de uma empresa, menor será a quantidade de emissões liberadas ao ambiente.

Destaca-se também da análise do ranking obtido que empresas de origem europeia apresentam uma tendência a produzir menos emissões que empresas oriundas de outros países, embora existam exceções.

Por fim, embora ainda existam limitações, destacando-se as relativas a carências na disponibilidade de informações dos relatórios do GRI, a aplicação do framework proposto apresentou potencial para efetuar avaliações e análises mais significativas do que simplesmente o reporte para fins de transparência pública das companhias de óleo e de gás.

\title{
FRAMEWORK DEVELOPMENT FOR RANKING ELABORATION AND ANALYSIS OF INTEGRATED OIL AND GAS COMPANIES SUSTAINABILITY REPORTS WITH GRI APPROACH
}

\begin{abstract}
The Global Report Initiative (GRI) is an international organization, non-profitable, that provides emission data and elaboration guidelines of sustainability reports for various industrial segments, such as the oil and gas sector. However, its limited to data presentation without any further analyzes being carried out aiming the production of more elaborated information as benchmark establishment. Therefore, in the present work, a own framework was proposed to organize the information in the elaboration of rankings of the integrated companies of oil and gas, verifying the existing relations between the production information and emissions of gases using a product-moment correlation. As a result, production and emission indicator rankings of integrated oil and gas companies were obtained. It is inferred from the study that the most productive companies in this energy segment are also the ones that cause the most emissions, and that companies of European origin tend to produce less emissions than companies from other countries. With the information analysis derived from the ranking obtained from the application of the proposed framework, it is possible for integrated oil and gas companies to more assertively plan their activities, aiming at their sustainable positioning.
\end{abstract}

Keywords: Gaseous emissions. Integrated oil and gas companies. Rankings. Global Report Initiative.

\section{REFERÊNCIAS}

ABREU, Ana Cristina Silva; ZARO, Elise Zoerger; LUIZ, Guilherme; VICENTE, Ernesto Fernandes Rodrigues; BELLEN, Hans Michael Van. Governança corporativa na estrutura conceitual do relato integrado: divulgações das empresas brasileiras participantes do projeto 
piloto. Revista de Gestão, Finanças e Contabilidade, Senhor do Bonfim, v. 6, n. 2, p. 31-49, 2016.

BENITES-LAZARO, Lira Luz; MELLO-THÉRY, Neli Aparecida. CSR as a legitimatizing tool in carbon market: Evidence from Latin America's Clean Development Mechanism. Journal of Cleaner Production,149, p. 218-226, 2017.

BENITES-LAZARO, Lira Luz; MELLO-THÉRY, Neli Aparecida. BG 2014 Sustainability Report. 2015. Disponível em: http://www.bg-group.com/495/sustainability/2014sustainability-report/. Acesso em: 14 dez. 2019.

BENITES-LAZARO, Lira Luz; MELLO-THÉRY, Neli Aparecida. BP 2014 Sustainability Review. 2015. Disponível em: http://www.bp.com/en/global/corporate/sustainability.html. Acesso em: 14 dez. 2019.

CHEN, Danfang; THIEDE, Sebastian; SCHUDELEIT, Timo; HERRMANN, Christoph. A holistic and rapid sustainability assessment tool for manufacturing SMEs. Manufacturing Technology, 63, p. 437-440, 2014.

CHEN, Danfang; THIEDE, Sebastian; SCHUDELEIT, Timo; HERRMANN, Christoph. CHEVRON Chevron Corporate Sustainability Report. 2015. Disponível em: http://www.chevron.com/corporateresponsibility/. Acesso em: 14 dez. 2019.

CHEN, Danfang; THIEDE, Sebastian; SCHUDELEIT, Timo; HERRMANN, Christoph. CNBC The value of sustainability reporting. 2016. Disponível em: http://gallery.cnbcafrica.com/video/?bctid=4233789191001. Acesso em: 14 dez. 2019.

HIGGINS, Colin.; COFFEY, Brian. Improving how sustainability reports drive change: a critical discourse analysis. Journal of Cleaner Production, 136, p. 18-29, 2016.

COHEN, Jacob; COHEN, Patricia; WEST, Stephan G.; AIKEN, Leona. Applied multiple regretion/correlation analysis for the behavioral sciences, 3. ed. Routledge, 2002, 736 p.

DOMINGUES, Ana Rita; LOZANO, Rodrigo; CEULEMANS, Kim; RAMOS, Tomás B. Sustainability reporting in public sector organisations: exploring the relation between the reporting process and organisational change management for sustainability. Journal of Environmental Management, v. 192, p. 292-301, 2017.

DOMINGUES, Ana Rita; LOZANO, Rodrigo; CEULEMANS, Kim; RAMOS, Tomás B.. ECOPETROL Sustainabilty Report 2014. 2015. Disponível em: http://www.ecopetrol.com.co/wps/portal/web_es/ecopetrol-web/our-company/PressRoom/management-and-sustainability-reports. Acesso em: 14 dez. 2015.

DOMINGUES, Ana Rita; LOZANO, Rodrigo; CEULEMANS, Kim; RAMOS, Tomás B.. ENI Annual Report 2014. 2015. Disponível em: http://www.eni.com/en_IT/investorrelation/investor_relations.shtml. Acesso em: 14 dez. 2015.

DOMINGUES, Ana Rita; LOZANO, Rodrigo; CEULEMANS, Kim; RAMOS, Tomás B. EUROPIA Contribution to EU Energy Pathways to 2050. 2011. Disponível em: http://www.europia.com . Acesso em: 20 abr. 2016. 
DOMINGUES, Ana Rita; LOZANO, Rodrigo; CEULEMANS, Kim; RAMOS, Tomás B. EXXON Corporate Citzenship Report 2014. 2015. Disponível em:

http://corporate.exxonmobil.com/en/community/corporate-citizenship-report. Acesso em: 14 dez. 2015.

FAYAD, Mohamed E.; SCHMIDT, Douglas C. JOHNSON, Ralph E. Building application frameworks: object oriented foundations of framework design. J. Wiley \& Sons, London, 688 p., 1999.

GJORUP, Ana Feital; FIDALGO, Elaine Cristina Cardoso; PRADO, Rachel Bardy; SCHULER, Azeneth Eufrasino. Análise de procedimentos para seleção de áreas prioritárias em programas de pagamento por serviços ambientais hídricos. Revista Ambiente e Água, Taubaté, v. 11, n. 1, p.225-238, 2016.

GOVONI, Darren. Java application frameworks. John Wiley \& Sons, New York, 1999. 432 p.

GOVONI, Darren. GRI Global Reporting Initiative. 2015. Disponível em: https://www.globalreporting.org/information/about-gri/Pages/default.aspx. Acesso em: 14 dez. 2015.

GOVONI, Darren. HESS 2014 Corporate Sustainability Report. 2015. Disponível em: http://www.hess.com/sustainability/sustainability-reports. Acesso em: 14 dez. 2015.

GOVONI, Darren. IPIECA/API/IOGP Oil and Gas Industry guidance on voluntary sustainability reporting. 2015. Disponível em: http://ipieca.org. Acesso em: 10 nov. 2015.

LIGTERINGEN, Ernest; ARBEX, Nelmara. GRI sustainability reporting: how valuable is the journey? Amsterdan, GRI Learning, 2012. 64 p

MARIMON, Frederic; ALONSO-ALMEIDA, María del Mar; RODRÍGUEZ, Martha del Pilar; ALEJANDRO, Klender Aimer Cortez . The worldwide diffusion of the global reporting initiative: what is the point? Journal of Cleaner Production, v.33, p.132-151, 2012.

MURADIAN, Roldán; CORBERA, Esteve; PASCUAL, Unai; KOSOY, Nicolas; MAY, Peter Herman. Reconciling theory and practice: an alternative conceptual framework for understanding payments for environmental services. Ecological Economics, v. 69, p. 12021208, 2009.

MURADIAN, Roldán; CORBERA, Esteve; PASCUAL, Unai; KOSOY, Nicolas; MAY, Peter Herman. OMV Sustainability Report 2014. 2015. Disponível em:

http://www.omv.com/portal/01/com/sustainability. Acesso em: 14 dez. 2015.

PERDAN, Slobodan; AZAPAGIC, Adisa. Carbon trading: current schemes and future developments. Energy Policy, v. 39, n. 10 p. 6040-6054, 2011.

PERDAN, Slobodan; AZAPAGIC, Adisa. PLATTS Platts Top 250. 2015. Disponível em http://top250.platts.com/Top250Rankings/2014/Region/IntegratedOilandGas. Acesso em: 27 nov. 2015. 
PERDAN, Slobodan; AZAPAGIC, Adisa. PETROBRAS Sustainability Report 2014. 2015. Disponível em: http://www.petrobras.com.br/en/society-and-environment/sustainabilityreport/. Acesso em: 14 dez. 2015.

RAHMAN, Syed Mahbubur; MIAH, Mohammad Dulal. The impact of sources of energy production on globalization: evidence from panel data analysis. Renewable and Sustainable Energy Reviews, v. 74, p. 110-115, 2017.

RAHMAN, Syed Mahbubur; MIAH, Mohammad Dulal. REPSOL Corporate Sustainability Report 2014. 2015 Disponível em:

http://www.repsol.com/es_en/corporacion/responsabilidad-corporativa/como-lohacemos/planes-sostenibilidad/. Acesso: $14 \mathrm{dez} 2015$.

SANTANA, Delano Mendes de; CASSIANO, Douglas Alves; LOURENÇO, Sérgio Ricardo. A energia como um reverso saliente no sistema sociotécnico do Brasil. Interfaces

Científicas: Humanas e Sociais, Aracaju, v. 3, n. 3, p. 113-122, 2015.

SANTANA, Delano Mendes de; CASSIANO, Douglas Alves; LOURENÇO, Sérgio Ricardo. SHELL Sustainability Report 2014. 2015. Disponível em:

http://reports.shell.com/sustainability-report/2014/servicepages/welcome.html. Acesso em: 14 dez. 2015.

SKJAERSETH, Jon Birger; WETTESTAND, Jorgen. Governance by EU emissions trading: resistance or innovation in the oil industry? International Environmental Agreements:

Politics, Law and Economics, v. 13 n. 1, p. 31-48, 2013.

SKJAERSETH, Jon Birger; WETTESTAND, Jorgen. STATOIL 2014 Sustainability

Report. 2015. Disponível em:

http://www.statoil.com/en/EnvironmentSociety/Sustainability/Pages/default.aspx. Acesso em: 14 dez. 2015.

SKJAERSETH, Jon Birger; WETTESTAND, Jorgen. SUNCOR 2014 Report on

Sustainability. 2015. Disponível em: http://www.suncor.com/en/responsible/1434.aspx. Acesso em: 14 dez. 2015.

SKJAERSETH, Jon Birger; WETTESTAND, Jorgen. TOTAL Corporate Social

Responsibility 2014. 2015. Disponível em: http://www.total.com/en/societyenvironment/CSR-Analysts. Acesso em: 14 dez. 2015.

VIGNEAU, L. et al. How do firms comply with international sustainability standards? processes and consequences of adopting the global reporting initiative. Journal of Business Ethics, v.131, p.469-482, 2015.

WANG, Qiang; LI, Rongrong. Research status of shale gas: a review. Renewable and Sustainable Energy Reviews, v. 74, p. 715-720, 2017. 


\section{DADOS DOS AUTORES}

\section{Ary Branco Adurens Júnior}

E-mail: adurens@gmail.com

Currículo Lattes: http://lattes.cnpq.br/9222428567454136

Mestrado em Energia pela Universidade Federal do ABC (UFABC), especialista em Marketing pela Fundação Getúlio Vargas (FGV). Atua profissionalmente em organizações na área comercial e na gestão de grandes contas e contratos e na assessoria, treinamento e representação no setor de óleo, gás e energia solar fotovoltaica. Possui interesse de pesquisa nos seguintes temas: Eficiência Energética, Planejamento e Gerenciamento de Energia, Sustentabilidade e Análise de Decisão Multicritério.

\section{Eduardo Christiano Cecone}

E-mail: eduardochristiano@hotmail.com

Currículo Lattes: http://lattes.cnpq.br/8374161318607164

Doutorado e mestrado em Energia pela UFABC, graduação em Engenharia de Materiais pelo Centro Universitário Fundação Santo André (CUFSA), especialista em Qualidade e Produtividade (UNIP), Engenheiro de Segurança do Trabalho (Unisant'anna) e Gestão de Projetos (Uniban). Conto com mais de 20 anos de experiência profissional, tendo ao longo dos últimos oito anos me dedicado exclusivamente às atividades de ensino, pesquisa e extensão, além da qualificação em nível de especialização, mestrado e doutorado.

\section{Chang Chung Wei}

E-mail: weichang@uol.com.br

Currículo Lattes: http://lattes.cnpq.br/0075438141553590

Mestrado em Energia pela UFABC, graduação em Engenharia Química pela Universidade Estadual de Campinas. Possui experiência na área de Industrial, com ênfase em adesivos industriais PSA, tratamento de resíduos sólidos industriais, processos químicos e projetos de eficiência energética.

\section{Fernando Gasi}

E-mail: fernando.gasi@ufabc.edu.br

Currículo Lattes: http://lattes.cnpq.br/8247613218944551

Pós-doutorado no Instituto Tecnológico da Aeronáltica (ITA), doutorado e mestrado Engenharia Química pela Universidade Estadual de Campinas (UNICAMP), graduação em Engenharia Mecânica pelo Centro Universitário da FEI. Possui vinte e cinco anos de experiência profissional no segmento industrial.

\section{Douglas Alves Cassiano}

E-mail: douglas.cassiano@ufabc.edu.br Currículo Lattes: http://lattes.cnpq.br/1987040329676323

Doutorado e mestrado em Engenharia Química pela UNICAMP e graduação em Engenharia Química pela Faculdade de Engenharia Industrial (FEI). Atualmente é professor Associado na Universidade Federal do ABC, UFABC. Atua na graduação nos cursos de Engenharia de Gestão, Engenharia de Energia e Bacharelado em Ciência e Tecnologia. Orientador de pesquisas no Programa de Pós Graduação em Engenharia de Produção e Energia da UFABC. 